\title{
6 Kritische Entscheidungssituationen und ethische Herausforderungen bei Notfällen in stationären und ambulanten Pflegeeinrichtungen
}

Thomas Luiz

\subsection{Bedeutung}

\subsubsection{Epidemiologie der Pflegebedürftigkeit}

Die zunehmende Alterung unserer Gesellschaft spiegelt sich direkt in der stetig steigenden Zahl Pflegebedürftiger wider: Ende 2013 bezogen in Deutschland 2,63 Millionen Menschen Leistungen der Pflegepflichtversicherung gemäß Sozialgesetzbuch (SGB) XI. Seit 1999 ist diese Zahl um mehr als 30\% gestiegen. Die tatsächliche Anzahl Pflegebedürftiger dürfte jedoch bereits heute 3 Millionen übersteigen: So werden ca. ein Drittel der Anträge auf ambulante und ca. jeder sechste Antrag auf stationäre Pflegeleistungen zunächst abgelehnt (Bundesministerium für Gesundheit 2013). Zudem beantragen nicht alle Betroffenen Leistungen der Pflegeversicherung. Infolge der stetigen Zunahme der Lebenserwartung wird 2030 mit bis zu 3,4 Millionen Pflegebedürftigen im Sinne des SCB XI gerechnet.

71\% der als pflegebedürftig Anerkannten werden zuhause gepflegt, davon zwei Drittel ausschließlich von ihren Angehörigen. Auf die enorme Belastung und praktische Bedeutung dieser Gegebenheit wird in Kapitel 6.6 eingegangen. 29\% der Pflegebedürftigen leben in Heimen (Statistisches Bundesamt 2013).

Die Höhe des individuellen Unterstützungsbedarfs wird im SGB XI anhand der Einstufung in sog. Pflegestufen ermittelt. 2013 fielen bei den zuhause Gepflegten $63 \%$ in Pflegestufe I, 29\% in Stufe II und 8\% (= ca. 150.000 Betroffene) in Stufe III. Bei den stationär Gepflegten war das Ausmaß der anerkannten Pflegebedürftigkeit deutlich höher: ca. 21\% gehörten der Stufe III an. 
6 Kritische Entscheidungssituationen und ethische Herausforderungen bei Notfällen in stationären und ambulanten Pflegeeinrichtungen

Unter den Pflegebedürftigen wiesen 35\% zusätzlich noch eine erheblich eingeschränkte Alltagskompetenz auf (Statistisches Bundesamt 2013). Umgangssprachlich wird hierbei oft von einer sog. „Pflegestufe o“ gesprochen. Die wichtigste Ursache dieser eingeschränkten Alltagskompetenz ist die Demenz. Einer aktuellen Untersuchung zufolge leidet mehr als die Hälfte der in stationären Einrichtungen Gepflegten unter dieser Erkrankung (Hoffmann 2014).

Betrachtet man die sog. Pflegequote, d.h. den Anteil Pflegebedürftiger in einer Altersgruppe, so steigt diese exponentiell von ca. $5,0 \%$ bei $70-75$-Jährigen über $21,0 \%$ bei 8o-85-Jährigen auf einen Spitzenwert von $64,4 \%$ bei den 9o-Jährigen und Älteren . Während bis ca. 75 Jahre die Pflegequote bei Männern die von Frauen übersteigt, kehrt sich dieses Verhältnis in höheren Altersgruppen um (Statistisches Bundesamt 2013).

\subsubsection{Bedeutung Pflegebedürftiger in Rettungsdienst und Krankentransport}

Angesichts des sehr fragilen Gesundheitszustandes Hochaltriger - ca. 80\% der über 70-Jährigen sind multimorbide (Prückner u. Madler 2009) - wundert es nicht, dass Pflegebedürftige im Rettungsdienst einen besonderen Stellenwert einnehmen: In einer früheren eigenen Untersuchung musste jeder fünfte Heimbewohner innerhalb eines Jahres notärztliche Hilfe in Anspruch nehmen, $15 \%$ davon sogar mehrfach. Beinahe 90\% der Patienten wurden in die Klinik eingewiesen (Luiz u. Madler 2009). Im eigenen Rettungsdienstbereich hat sich zudem während der letzten 10 Jahre die Rate an Notarzteinsätzen in Altenheimen verdoppelt und liegt derzeit bei ca. 10\% aller Notarzteinsätze. Bei den Krankentransporten haben sogar 30\% ein Altenheim als Einsatzort oder Transportziel (bislang unveröffentlichte eigene Daten).

In Deutschland sind ca. 3 Millionen Menschen pflegebedürftig. Von diesen werden ca. $70 \%$ zuhause gepflegt, davon wiederum ca. zwei Drittel ausschließlich durch ihre Angehörigen. Pflegebedürftige Patienten nehmen den Rettungsdienst weit überdurchschnittlich häufig in Anspruch: Sie indizieren je nach Rettungsdienstbereich bis zu 10\% der Notarzteinsätze und bis zu 30\% der Krankentransporte.

\subsection{Allgemeine ethische Grundsätze und grundsätzliche Heraus- forderungen bei der Versorgung pflegebedürftiger Patienten im Rettungsdienst}

Die allgemein anerkannten ethischen Prinzipien des Umgangs mit Patienten (Beauchamp u. Childress 2012; Lippert et al. 2010) stellen bei der Versorgung pflegebedürftiger Patienten im Rettungsdienst eine besondere Herausforderung dar:

- Pflicht zur Fürsorge

- Vermeidung von Schaden

- Wahrung der Gerechtigkeit

- Wahrung der Würde

- Aufrichtigkeit in der Kommunikation

Fürsorge: Die Anamneseerhebung und Untersuchung pflegebedürftiger Notfallpatienten sind u.U. deutlich schwieriger und zeitaufwendiger als bei jüngeren Patienten. 
Es steht jedoch außer Frage, dass diese Patienten, ungeachtet der aktuellen Einsatzbelastung, mit der dem jeweiligen Krankheitsbild und Zustand angemessenen Sorgfalt versorgt werden müssen: Ein 83-jähriger Demenzkranker, der im Altenheim auf der Treppe schwer gestürzt ist und dessen aktuelle Orientiertheit und Vigilanz mangels Kenntnis des Vorbefunds nicht klar einschätzbar sind, muss daher vom Notarzt im Zweifel für eine dringliche CT-Untersuchung in die Klinik begleitet werden. Eine weitere Verpflichtung zur Fürsorge betrifft die Frage der aktuellen Versorgungssituation:

Werden konkrete, bislang nicht erkannte bzw. nicht adäquat behandelte medizinische Risiken oder eine relevante Schädigung infolge einer ungenügenden pflegerischen Versorgung festgestellt, müssen die Pflegenden darauf aufmerksam gemacht und ggf. weitere Schritte zum Schutz des Patienten eingeleitet werden.

Je nach Situation kann dies eine schriftliche Anweisung an das Pflegepersonal und/ oder eine Kontaktaufnahme mit dem Hausarzt sein (z.B. bei schwerer oder wiederholter Hypoglykämie infolge unzureichender Blutzuckerüberwachung eines insulinpflichtigen Diabetikers). Bei massiven Komplikationen kann sogar eine umgehende Einweisung in die Klinik indiziert sein, z.B. im Falle eines tiefreichenden Dekubitus, der bei einem Einsatz wegen einer ,akuten schweren Atemnot“, die sich jedoch vor Ort suffizient therapieren ließ, als „Nebenbefund“ entdeckt wird.

Schadensvermeidung:Viele Erkenntnisse der modernen Medizin wurden nicht an pflegebedürftigen, hochaltrigen Menschen, sondern unter den sehr viel restriktiveren Bedingungen kontrollierter klinischer Studien gewonnen. Ihr medizinischer Nutzen ist für diese Population schlechter belegt, die Risiken sind jedoch erhöht (Benetos et al. 2015).

\section{Alle Maßnahmen, insbesondere die Gabe von Medikamenten mit hohem Ri- siko von Neben- und Wechselwirkungen, z.B. Antiarrhythmika oder gerin- nungshemmende Präparate, sind besonders sorgfältig abzuwägen.}

Auch die Indikation zur Klinikeinweisung, z.B. nach einer Synkope oder hypertensiven Entgleisung, ist in jedem Einzelfall sorgfältig abzuwägen: Überwiegen die Vorteile einer postulierten intensiveren medizinischen Betreuung die Probleme, die durch die u.U. schmerzhafte Umlagerung und Fahrt in die Klinik entstehen? Wird das bei multimorbiden alten Menschen deutlich erhöhte Delirrisiko durch Verlust der vertrauten Umgebung beachtet? Werden Hör-, Seh- und Gehhilfen des Patienten mit in die Klinik genommen, um das Delirrisiko zu senken? Wäre eine etwas längere notärztliche Verweildauer vor Ort oder ein nachfolgender Kontrollbesuch des Ärztlichen Bereitschaftsarztes eine geeignete Alternative?

Gerechtigkeit: Pflegebedürftige Patienten müssen dieselben Chancen auf Zugang zu akutmedizinischen Ressourcen erhalten wie andere Patienten. Entscheidend muss die Dringlichkeit des Zustands sein, nicht der Einsatzort. Konkret darf der Hausbesuch 
6 Kritische Entscheidungssituationen und ethische Herausforderungen bei Notfällen in stationären und ambulanten Pflegeeinrichtungen

des Ärztlichen Bereitschaftsdienstes wegen eines akuten Abdomens oder der Einsatz des Notarztes wegen eines Lungenödems im Altenheim keine geringere Priorität erhalten als dasselbe Krankheitsbild bei einem 48-jährigen, bislang gesunden Patienten.

Würde: Es sollte eine Selbstverständlichkeit sein, dass sich der Arzt bzw. Rettungsassistent immer, auch bei dementen Patienten, mit Namen, Funktion und Grund des Hierseins vorstellt und alle geplanten Maßnahmen ankündigt bzw. um die entsprechende Erlaubnis bittet. Dies betrifft insbesondere schmerzhafte Maßnahmen sowie das Entkleiden. Liegt der Patient hilflos in seinem Erbrochenen oder seinen Exkrementen, bedarf er besonderer Zuwendung. Häufig ist es nach entsprechender Absprache möglich und für den Betroffenen eine große Erleichterung, wenn die ihm vertraute Pflegekraft vor dem Transport zumindest eine Grobreinigung und einen Wechsel des Nachthemds bzw. Pyjamas vornimmt, während die Einsatzkräfte in der Zwischenzeit z.B. vor dem Zimmer die Trage für den Transport vorbereiten, Medikamentenlisten sichten, etc.

Aber auch die Würde von Mitbewohnern ist zu beachten: Was empfindet ein ans Bett gefesselter, u.U. dementer Mensch, wenn sein Zimmernachbar reanimiert wird? Wie kann er das „überfallartige“ Eindringen von bis zu 5 rot gekleideten Personen, das Rufen von martialisch anmutenden Kommandos, das Aufschneiden von Kleidung mit der Rettungsschere, die Alarmsignale des Defibrillators etc. verarbeiten?

Aufrichtigkeit in der Kommunikation: Mit den vor Ort bestehenden eingeschränkten Möglichkeiten besteht häufig Unklarheit über die Prognose einer plötzlichen Erkrankung. Dies sollte bewusstseinsklaren Patienten, aber auch Angehörigen gegenüber klar geäußert werden. Gleichzeitig sollte jedoch auch ein für den Notarzt erkennbarer (prä) finaler Zustand mit dem nötigen Einfühlungsvermögen im Hinblick auf die Angemessenheit weiterer Maßnahmen (palliative häusliche oder klinische Versorgung, spirituelle Begleitung etc.) angesprochen werden.

Einsätze in Altenheimen bedürfen vor allem Geduld und eines bedachtsamen Abwägens von Nutzen und Risiken. Häufig sind es scheinbare „Kleinigkeiten“ und eingeschliffene Unachtsamkeiten, welche die Würde des pflegebedürftigen Patienten verletzen. Ein Perspektivwechsel kann sehr hilfreich sein, um nicht nur medizinische, sondern auch emotionale Bedürfnisse des Patienten besser zu erkennen.

\subsection{Wahrung der Patientenautonomie und ihre Grenzen}

\subsubsection{Grundsätze}

Unzweifelhaft haben auch Pflegebedürftige das Recht, über Zeitpunkt, Art, Ort und Ausmaß medizinischer Maßnahmen selbst zu bestimmen. Entsprechend sind eindeutige, aktuelle, verbale oder auf den aktuellen Fall anwendbare frühere schriftliche Willensbekundungen rechtlich bindend.

Bei der präklinischen Versorgung pflegebedürftiger Notfallpatienten besteht die besondere Problematik nun darin, dass ein erheblicher Anteil aktuell entscheidungs- bzw. kommunikationsunfähig ist. Gleichzeitig sind schriftliche Willens- 
bekundungen aufgrund der Dringlichkeit nicht rechtzeitig genug zugänglich (z.B. Arztbriefe, Gespräch mit dem Hausarzt) oder in der aktuellen Situation nicht eindeutig interpretier- und anwendbar. Wenig brauchbar ist z.B. eine Patientenverfügung mit dem Inhalt, dass im Falle eines unumkehrbaren Verlusts des Bewusstseins keine invasiven Maßnahmen getroffen werden sollen. Eine für den Rettungsdienst sehr gut geeignete, in Mainz entwickelte Formulierungshilfe bei Palliativpatienten zeigt Abbildung 5 .

\section{„P A L M A“}

Patienten- Anweisungen für lebenserhaltende Maßnahmen - für Patienten in einer palliativen Situation ergänzend zur ausführlichen Patientenverfügung -

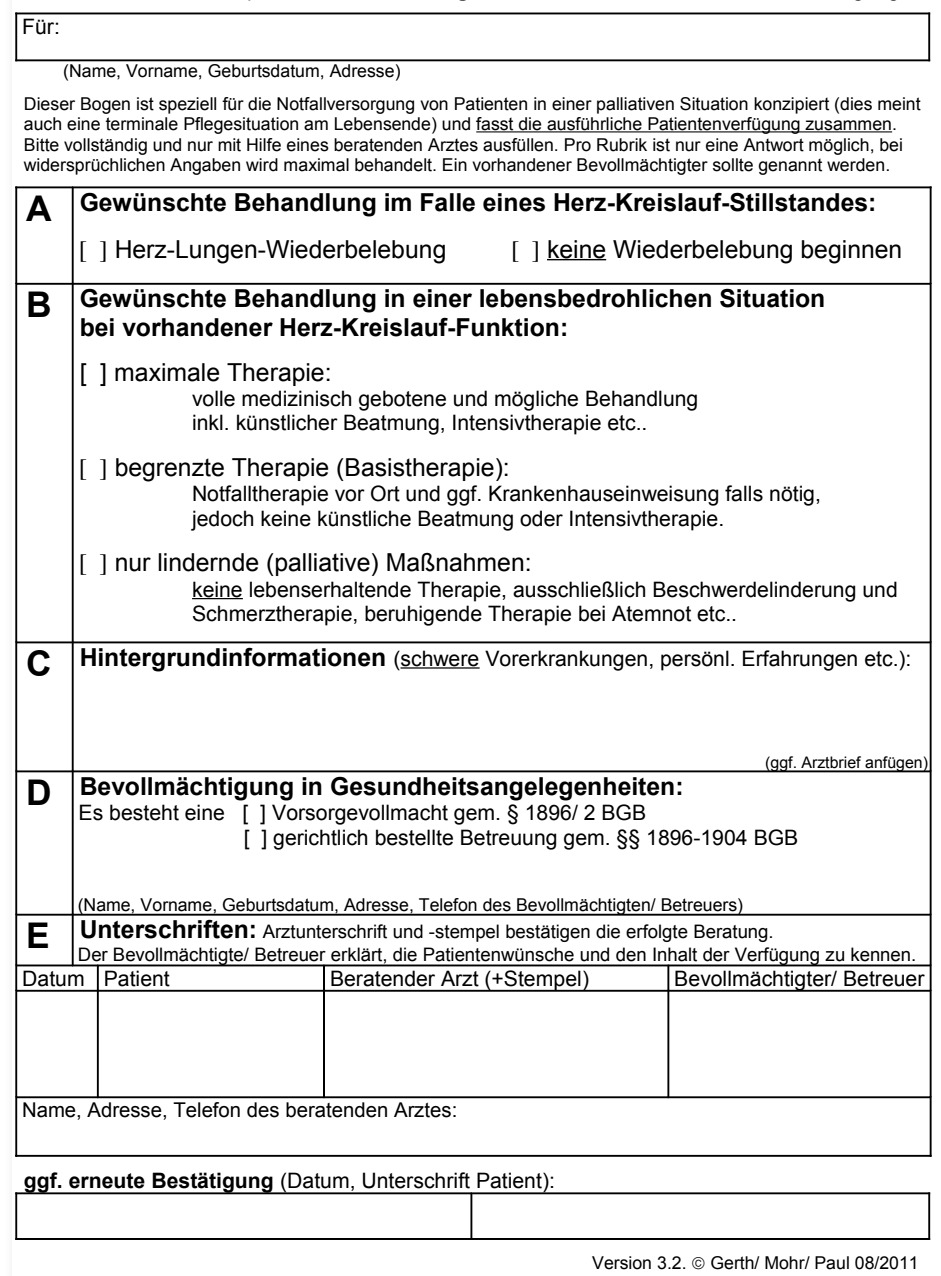

Abb. 5 „PA L M A“: Patientenanweisungen für lebenserhaltende Maßnahmen für Patienten in einer palliativen Situation ergänzend zur ausführlichen Patientenverfügung (Abdruck mit freundlicher Genehmigung von Dr. Mathias Gerth, Universitätsmedizin Mainz) 
Eine zentrale Problematik besteht darin, ad hoc bei einem bewusstlosen pflegebedürftigen Patienten, dessen umfangreiche medizinische und soziale Biographie dem Notarzt völlig unbekannt sind, abschätzen zu müssen, ob noch eine kurative oder aber eine palliative Zielsetzung, oder u.U. sogar ein Verzicht auf sämtliche medizinischen Maßnahmen angebracht ist. Die Unterbringungssituation in einem Pflegebett und der körperliche Zustand (z.B. Kontrakturen, Ernährungszustand) machen zwar evident, dass es sich um eine schwerste Pflegebedürftigkeit handelt. Bedauerlicherweise beinhalten die medizinischen Daten in den Pflegeakten häufig nur die Angabe von Diagnosen und Dauermedikation, jedoch keine konkreten aktuellen Angaben zu Erkrankungsstadien, Prognose etc.

Untersuchungen zeigen zudem, dass sich die Einstellung von mental kompetenten Altenheimbewohnern zu Fragen wie Wiederbelebung oder künstliche Ernährung im Laufe der Zeit in nicht unerheblicher Weise ändern kann (McParland et al. 2003). Damit ihr aktueller Wille im Notfall angemessen berücksichtigt werden kann, müssen sich Betroffene somit regelmäßig aktiv mit diesen Fragen beschäftigen (bzw. darauf behutsam angesprochen werden), sich gegenüber den Pflegenden eindeutig äußern bzw. ggf. bestehende Patientenverfügungen ändern. Dies ist hierzulande nur selten der Fall, sodass der Rettungsdienst häufig keine wirklich verlässlichen Entscheidungshilfen zur Hand hat.

In Kliniken gängige Instrumente wie Ethikkonsile, die bei solchen Situationen eines Entscheidungsnotstands Hilfestellung leisten könnten, sind präklinisch nicht verfügbar (s. Tab. 10).

Die präklinische Notfallmedizin und die innerklinische Intensivmedizin unterscheiden sich hinsichtlich der Rahmenbedingungen der Entscheidungsfindung ganz wesentlich und das Risiko von Fehlentscheidungen ist erheblich.

\subsubsection{Der bewusstlose Patient}

Vor dem Hintergrund der rechtlichen Forderung, im Zweifel umgehend lebensrettende Maßnahmen einzuleiten (Wiese et al. 2009), wird bei einem Ausfall der Vitalfunktionen, der nicht eindeutig, z.B. anhand eines aktuellen Arztbriefs, auf einen Terminalzustand eines inkurablen Leidens zurückzuführen ist, bei entscheidungsunfähigen Patienten auch bei Vorliegen einer Pflegebedürftigkeit zunächst mit invasiven Maßnahmen begonnen, bis ggf. weitere Informationen eine anderslautende Entscheidung, z.B. den Abbruch einer Reanimation, rechtfertigen (s. Fallbeispiel in Kap. 6.5).

Aktuelle Willensbekundungen des entscheidungsfähigen Patienten sowie auf den aktuellen Zustand anwendbare Patientenverfügungen sind zu beachten. Glaubhafte mündliche Überlieferungen des Willens nicht ansprechbarer Patienten sollten vor Zeugen möglichst schriftlich dokumentiert und beachtet werden. Liegen widersprüchliche Angaben oder überhaupt keine Erkenntnisse zum Patientenwillen vor, muss die für den Patienten mutmaßlich beste medizinische Entscheidung getroffen werden. Dies bedeutet beim Ausfall von Vitalfunktionen, im Zweifel zunächst mit Wiederbelebungsmaßnahmen zu beginnen. 
Tab. 10 Unterschiede zwischen innerklinischer Intensiv- und präklinischer Notfallmedizin

\begin{tabular}{|c|c|c|}
\hline & Intensivmedizin & Präklinische Notfallmedizin \\
\hline $\begin{array}{l}\text { Verfügbarkeit schriftlicher } \\
\text { Willensbekundungen } \\
\text { des Patienten (Patienten- } \\
\text { verfügung, Betreuungs- } \\
\text { verfügung, Vorsorgevollmacht) }\end{array}$ & $\begin{array}{l}\text { ggf. im Verlauf des Aufenthalts } \\
\text { verfügbar }\end{array}$ & $\begin{array}{l}\text { häufig nicht oder nicht sofort } \\
\text { verfügbar }\end{array}$ \\
\hline $\begin{array}{l}\text { Interpretation schriftlicher } \\
\text { Willensbekundungen }\end{array}$ & $\begin{array}{l}\text { durch ausführliche Gespräche mit } \\
\text { Angehörigen und/oder Hausarzt } \\
\text { häufig möglich }\end{array}$ & unter Zeitdruck häufig nicht eindeutig \\
\hline $\begin{array}{l}\text { genaue Kenntnis der } \\
\text { Patientenbiographie }\end{array}$ & $\begin{array}{l}\text { abhängig von früheren Aufenthalten } \\
\text { bzw. der Dauer des Aufenthalts } \\
\text { sowie Auskünften Angehöriger } \\
\text { und/oder des Hausarztes }\end{array}$ & $\begin{array}{l}\text { nur ausnahmsweise gegeben (z.B. } \\
\text { Kenntnis des Patienten aus früheren } \\
\text { Rettungsdiensteinsätzen oder } \\
\text { aufgrund der Dualfunktion als Notarzt } \\
\text { und zugleich Haus- oder Klinikarzt von } \\
\text { Patienten) }\end{array}$ \\
\hline $\begin{array}{l}\text { Prognoseabschätzung bezüglich } \\
\text { der aktuellen Erkrankung }\end{array}$ & $\begin{array}{l}\text { direkt nach Aufnahme häufig noch } \\
\text { unvollständig; } \\
\text { im Falle früherer Aufenthalte oder } \\
\text { im Verlauf eines längeren } \\
\text { Aufenthalts: im Vergleich zur } \\
\text { Präklinik sehr viel umfassendere und } \\
\text { präzisere Informationsgrundlage }\end{array}$ & $\begin{array}{l}\text { besonders bei zeitkritischen } \\
\text { Situationen häufig nicht verlässlich } \\
\text { möglich }\end{array}$ \\
\hline $\begin{array}{l}\text { Kenntnis von die Prognose } \\
\text { beeinflussenden Vor- und } \\
\text { Begleiterkrankungen }\end{array}$ & $\begin{array}{l}\text { direkt nach Aufnahme häufig } \\
\text { unvollständig; } \\
\text { im Falle früherer Aufenthalte oder } \\
\text { im Verlauf eines längeren } \\
\text { Aufenthalts: häufig umfassend }\end{array}$ & $\begin{array}{l}\text { besonders bei zeitkritischen } \\
\text { Situationen häufig nicht gegeben } \\
\text { selten Vorliegen aktueller Arztbriefe } \\
\text { oder direkter telefonischer Kontakt mit } \\
\text { Hausarzt oder sonstigen behandeln- } \\
\text { den Ärzten }\end{array}$ \\
\hline $\begin{array}{l}\text { diagnostisches und therapeuti- } \\
\text { sches Repertoire bei Ausfall von } \\
\text { Vitalfunktionen }\end{array}$ & $\begin{array}{l}\text { abhängig von Versorgungsstufe: } \\
\text { umfassende Bildgebung, } \\
\text { Organersatzverfahren }\end{array}$ & $\begin{array}{l}\text { stark eingeschränkt: } \\
\text { Diagnostik: EKG, Pulsoxymetrie, } \\
\text { Temperatur; selten Point-of-Care-Labor } \\
\text { und Ultraschall } \\
\text { Therapie: Beatmung, Katecholamine, } \\
\text { Defibrillation, Thoraxkompression }\end{array}$ \\
\hline $\begin{array}{l}\text { konsiliarische Hinzuziehung } \\
\text { weiterer medizinischer } \\
\text { Experten }\end{array}$ & $\begin{array}{l}\text { je nach Versorgungsstufe evtl. } \\
\text { eingeschränkt }\end{array}$ & in der Regel nicht möglich \\
\hline $\begin{array}{l}\text { Beratung in ethischen } \\
\text { Fragestellungen (Ethikkomitee, } \\
\text { Ethikberatung, Ethikkonsil) }\end{array}$ & $\begin{array}{l}\text { häufig ja, mit Ausnahme akuter } \\
\text { Entscheidungsnot direkt bei der } \\
\text { Klinikaufnahme }\end{array}$ & im Einsatz nicht möglich \\
\hline
\end{tabular}

\subsubsection{Der demente Patient}

Eine fortgeschrittene Demenz wird hierzulande immer noch häufig mit dem kompletten Verlust der Selbstbestimmungsfähigkeit gleichgesetzt. Die Rechte Betroffener werden dann im Sinne einer sog. „substituierten Entscheidungsfindung “ durch Drit- 
te (Betreuer, Gericht) wahrgenommen. Dieses Vorgehen verkennt, dass die Fähigkeit zur Selbstbestimmung keine fixe „Ja-Nein“-Situation darstellt. Demenzkranke können je nach Situation und Zeitpunkt durchaus ihre Bedürfnisse und ihren Willen ausdrücken, wenn sie in ihrer Kommunikation angemessen unterstützt werden. Die o.g. starre Praxis wird daher zunehmend kritisch gesehen und die Notwendigkeit eines individuelleren Vorgehens betont (Birnbacher et al. 2015).

Bei dementen Patienten entbindet ein Betreuungsverhältnis den Behandelnden nicht von der Pflicht, zu versuchen, den aktuellen Willen des Betroffenen zu eruieren. Neben verbalen Äußerungen ist auf nonverbale Hinweise zu achten, wie z.B. das konsequente Verschließen des Mundes bei der Nahrungsaufnahme trotz wiederholter geduldiger Ermunterung und Eingehen auf spezielle Essgewohnheiten, oder das vehemente, wiederholte Wegziehen des Armes bei der hinreichend erklärten venösen Kanülenanlage. Unter Umständen finden sich hier Alternativen, z.B. eine s.c.- anstelle einer i.v.-Injektion oder eine Kurzinfusion mit früher Entfernung der Verweilkanüle anstelle einer den Patienten ängstigenden und immobilisierenden längerdauernden Infusion.

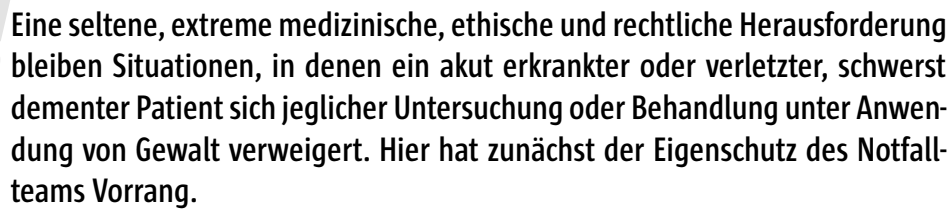

Gleichzeitig sollte versucht werden, vertraute Bezugspersonen zu involvieren, um mit deren Hilfe den Patienten zu beruhigen. Scheitert dies und besteht eine akute Eigen- oder Fremdgefährdung fort, sollten die Vollzugsbehörden eingeschaltet werden. Körperliche und/oder pharmakologische Zwangsmaßnahmen sind jedoch auf ein Minimum zu beschränken. Falls eine Einweisung in eine allgemeine oder psychiatrische Klinik notwendig ist, sollten Bezugspersonen den Betroffenen möglichst begleiten oder frühzeitig besuchen, um die psychischen Auswirkungen der Zwangsbehandlung zu minimieren und die Reorientierung zu erleichtern.

Bei dementen Patienten muss die Fähigkeit zur Kommunikation und Selbstbestimmung individuell geprüft werden. Eine vorliegende Betreuung ersetzt diese Verpflichtung nicht. Angesichts der hohen Prävalenz der Erkrankung sind intensivere Schulungen des Rettungsdienstes zum Umgang mit dementen Patienten dringend notwendig.

\section{Fallbeispiel:}

Der Notarzt wird in ein Altenheim zu einer 81-jährigen bewusstseinsgetrübten Patientin angefordert. Die Patientin ist seit 5 Tagen bei Z.n. Implantation einer Hüfthemiprothese in Kurzzeitpflege. Es bestehen ein gut eingestellter Diabetes mellitus, ein Hypertonus sowie ein chronisches Schmerzsyndrom bei ausgeprägter Osteoporose und Polyarthrose. Die Patientin sei seit ihrer Aufnahme immer apathischer geworden. Der aktuelle Blutzucker betrage 
156 mg\%. Der Ärztliche Bereitschaftsdienst habe am Vortag wegen hypotoner Blutdruckwerte die Dosis der Antihypertensiva reduziert und die Bedarfsmedikation mit Metamizol abgesetzt. Der Zustand habe sich seit heute früh allerdings weiter verschlechtert. Die Patientin erhalte keine weiteren Medikamente.

Befund: Die Atmung ist langsam und unregelmäßig, die Atemwege sind intermittierend verlegt, die Sauerstoffsättigung beträgt $80 \%$. Der Puls ist verlangsamt und arrhythmisch, der Blutdruck beträgt $70 / 50 \mathrm{mmHg}$, die Temperatur $35,1^{\circ} \mathrm{C}$. Die Patientin reagiert nur mit trägen, ungezielten Abwehrbewegungen der linken Hand auf Schmerz. Die Muskeleigenreflexe sind beidseits abgeschwächt, ein Meningismus liegt nicht vor. Die Pupillen sind beidseits relativ eng, die Pupillenreaktion ist träge. Anamnestisch und klinisch liegen keine Hinweise auf ein Trauma vor.

Als Sofortmaßnamen werden die Atemwege freigemacht und Sauerstoff appliziert. Außerdem wird die Intubation vorbereitet und über eine Venenverweilkanüle Vollelektrolytlösung infundiert. Zusätzlich erhält die Patientin fraktioniert $2 \mathrm{ml}$ eines milden Vasopressors. Die Vitalwerte stabilisieren sich daraufhin leicht. Die Leitstelle wird hinsichtlich einer instabilen intubationspflichtigen Patientin mit Verdacht auf Stammhirninfarkt bzw. -blutung informiert. In diesem Moment legt die Altenpflegerin eine aktuelle Patientenverfügung vor, in dem für den Fall einer Verschlechterung des Gesundheitszustands eine Wiederaufnahme in die Klinik und speziell eine künstliche Beatmung strikt abgelehnt werden. Der Notarzt bittet daher, dringlich die Angehörigen anzurufen, um das weitere Vorgehen zu besprechen, und fährt währenddessen mit der klinischen Untersuchung fort. Dabei fallen am Rücken 2 Fentanyl-Pflaster auf. Diese Medikation war der Pflegekraft nicht bekannt. Mittlerweile besteht Telefonkontakt mit den Angehörigen. Dabei wird deutlich, dass die Fentanyl-Gabe dem Altenheim irrtümlich nicht mitgeteilt worden war. Zudem hatten die Angehörigen am Vortag, nachdem sie erfahren hatten, dass das Metamizol abgesetzt worden war, aus Sorge vor einem Schmerzdurchbruch selbständig ein weiteres Fentanylpflaster aufgeklebt. Bei Verdacht auf eine Opiatüberdosierung wird nun Naloxon verabreicht, woraufhin die Patientin rasch aufklart. Nach kurzer Erläuterung der Situation stimmt sie einem Kliniktransport bis zur völligen Stabilisierung ihres Zustands zu. Am Folgetag erfolgt bei relativem Wohlbefinden die Rückverlegung ins Heim.

\subsection{Sterbende Patienten}

Der Tod ist im höchsten Lebensalter ein jederzeit erwartbares Ereignis oder sogar ein herbeigesehnter Endpunkt eines austherapierten Leidens. Dennoch wird bei Sterbenden heute häufig der Rettungsdienst alarmiert. Die Gründe hierfür sind äußerst vielfältig: fehlende Kenntnis des Patientenwillens, Nichtbefolgen vorliegender Patientenäußerungen oder -verfügungen, Unkenntnis oder fehlende Akzeptanz des Sterbeprozesses, medizinische, physische, psychische oder zeitliche Überforderung der Pflegenden (z.B. bei sehr hohem Pflegeaufwand, starken Schmerzen oder massiver Luftnot), die irrige Annahme, der Sterbeprozess müsse von einem Arzt begleitet oder „erlaubt“ werden, eine schwindende Bereitschaft niedergelassener Ärzte zu Hausbesuchen, die unzureichende Verfügbarkeit von Palliativdiensten, die fehlende Verfügbarkeit von Betäubungsmitteln bei niedergelassenen Ärzten und Ärzten im Bereitschaftsdienst, häufig jedoch auch „nur“ der Wunsch nach Abgabe der „letzten Verantwortung“ (Luiz u. Madler 2009). 
Viele dieser Einsätze wären bei besserer Vorbereitung und Aufklärung und der Verfügbarkeit anderer Institutionen vermeidbar. Insbesondere ambulante Palliativdienste sind bislang vorwiegend auf Ballungsräume konzentriert und/oder nur selten rund um die Uhr einsatzbereit. Noch seltener ist eine spezialisierte ambulante Palliativversorgung (SAPV) verfügbar, die jedoch den Rettungsdienst wirksam entlasten könnte (Wiese et al. 2010).

Ist im konkreten Fall der Rettungsdienst bereits vor Ort bzw. ist kein spezialisierter Palliativdienst verfügbar, ist zunächst zu prüfen, welches Problem im Vordergrund steht: eine konkrete medizinische Symptomatik, die ärztliche Maßnahmen erfordert, ein pflegerisches Problem oder die emotionale Überlastung der Angehörigen.

\section{Ein würdevolles Sterben ist nur möglich, wenn quälende Symptome wie star- ke Schmerzen, Luftnot, Durst oder heftiges Erbrechen suffizient behandelt werden.}

Diese Symptome sind häufig (Brandt et al. 2005), lassen sich jedoch mit den im Notarztdienst verfügbaren Mitteln oftmals gut beherrschen: Dyspnoische Patienten sollten mit erhöhtem Oberkörper gelagert werden. Ein terminales Lungenödem lässt sich durch fraktionierte Morphingabe häufig suffizient lindern. Inwieweit zusätzlich Sauerstoff sinnvoll ist bzw. vom Patient toleriert wird, ist situationsabhängig zu prüfen.

\section{Eine Intubation ist bei einem erkennbaren Sterbeprozess weder medizinisch noch ethisch oder rechtlich vertretbar.}

Schmerzdurchbrüche lassen sich häufig durch die titrierte i.v.-Gabe von Opiaten, ggf. in Kombination mit Metamizol oder Paracetamol, beherrschen.

Sterbende stellen häufig das Trinken und Essen ein. Bei einem Großteil der Patienten ergibt sich hieraus kein Handlungsbedarf. Je nach Situation wird aber das Anfeuchten der Lippen oder der Mundschleimhäute als angenehm empfunden. Eine routinemäßige Hydrierung durch Infusionen hat weder einen Einfluss auf Symptome wie Schwäche, Muskelkrämpfe oder Halluzinationen, noch auf die Dauer der Sterbephase. Angehörige, z.T. auch die Sterbenden selbst, empfinden jedoch eine aktive Flüssigkeitszufuhr häufig als angenehm oder trostreich, bzw. das Unterlassen dieser Maßnahme u.U. als belastend (Parry et al. 2013). Sollte der Patient schluckunfähig sein und starken Durst empfinden, kann daher eine s.c.-, im Einzelfall auch eine kurzzeitige i.v.-Flüssigkeitssubstitution angezeigt sein.

Sofern eine Weiterbehandlung durch den Ärztlichen Bereitschaftsdienst oder Hausarzt nötig und möglich ist, sollten diese telefonisch informiert und eine Kopie des Einsatzprotokolls vor Ort belassen werden. Falls ein anderer Arzt jedoch nicht rasch genug verfügbar ist oder dieser nicht die notwendigen Medikamente wie z.B. Opiate vorhält, ist auch ein erneuter Notarzteinsatz gerechtfertigt. 
Der Notarzt sollte mit den Angehörigen bzw. dem Pflegepersonal stets das weitere konkrete Vorgehen besprechen, dieses in der Pflegeakte notieren und Nachbehandelnde informieren. Bei Nichtverfügbarkeit anderer Dienste ist ein Notarzteinsatz beim Sterbenden zur Linderung quälender Symptome gerechtfertigt.

\section{Fallbeispiel:}

Der Notarzt wird von der Besatzung eines RTW zu einer 73-jährigen Patientin mit schwerem Schockzustand nachgefordert. Die stark exsikkierte, kachektische, an Multipler Sklerose leidende Patientin hatte nach Angaben des Ehemannes seit Wochen stetig weniger gegessen und getrunken und war zuletzt kaum mehr ansprechbar. Er hatte den Ärztlichen Bereitschaftsdienst informiert, der eine schwere Pneumonie mit beginnender Sepsis diagnostizierte und die Patientin umgehend mittels RTW in die Klinik einwies. Die RTW-Besatzung stellte beim Eintreffen eine massive Beeinträchtigung aller Vitalfunktionen fest. Aufgrund fehlender Transportfähigkeit sei die Alarmierung des Notarztes erfolgt und Sauerstoff appliziert worden. Bei der orientierenden Untersuchung durch den Notarzt ist die Patientin komatös, tachypnoisch und zyanotisch. Es bestehen pneumonieverdächtige Rasselgeräusche mit Sekretstau, die Temperatur liegt tympanal bei $35,4^{\circ} \mathrm{C}$. Ein vorliegender Entlassbrief einer Klinik bestätigt die schwerste Grunderkrankung. Der Gatte berichtet, er pflege seine Ehefrau seit 6 Jahren. Ihr Gesundheitszustand habe sich stetig bis zur völligen Immobilisation verschlechtert. In einer dem Notarzt vorgelegten Patientenverfügung hat die Frau festgelegt, sie wolle im Falle einer weiteren Verschlechterung nicht in die Klinik, sondern zuhause „in Frieden sterben“. Der Mann äußert, er wolle zwar den Willen seiner Frau respektieren, könne sie jedoch andererseits „nicht einfach ersticken lassen“. Gleichzeitig habe er tiefe Sorge, dass seine Frau vom Rettungsdienst in die Klinik gebracht und dort auf einer Intensivstation „künstlich am Leben" gehalten werde.

Der Notarzt erläutert dem Gatten die Intention einer palliativen Versorgung, die sowohl dem Wunsch der Patientin als auch dessen Sorge gerecht werden kann. Der Ehemann nimmt dies mit größter Erleichterung auf. Nach Gabe von $3 \mathrm{mg}$ Morphinsulfat und vorsichtigem Absaugen von viel eitrigem Trachealsekret bessert sich die Atmung bereits spürbar. Leider ist kein Pflegedienst, der die weitere Versorgung akut übernehmen könnte, erreichbar. Eine häusliche Betreuung durch den Ehemann oder Anverwandte scheidet ebenfalls aus. Nach Abwägung aller Aspekte wird die Patientin daraufhin in Begleitung des Ehemanns unter telefonischer Voranmeldung der palliativen Zielsetzung in eine Klinik transportiert, wo sie unter Fortführung der Sauerstoffgabe und Morphintherapie einige Stunden später ruhig entschläft.

Ärzte und Rettungsfachpersonal müssen auf Situationen vorbereitet werden, in denen es nicht mehr darum geht, Leben zu retten, sondern Sterbenden mit palliativen Maßnahmen beizustehen. Ein Transport in die Klinik sollte auf diejenigen Situationen beschränkt bleiben, in denen der Arzt zur Erkenntnis gelangt, dass dem Sterbenden ein würdevoller Tod im häuslichen Umfeld bzw. Heim nicht möglich ist. 


\subsection{Kreislaufstillstand}

Die Anzahl an Reanimationen in Altenheimen steigt weltweit. So erfolgt inzwischen etwa in Japan jede fünfte (Kitamura et al. 2014), in Kopenhagen, Dänemark, etwa jede zehnte (Soholm et al. 2014) und in Melbourne, Australien (Deasy et al. 2011), etwa jede zwölfte Reanimation in Altenheimen.

Die Prognose ist insgesamt sehr ungünstig: Die meisten Studien geben Entlassraten aus der Klinik von nur 1-4\% und einen hohen Anteil neurologisch sehr ungünstiger Verläufe an (Deasy et al. 2011; Kitamura et al. 2014). Dies korreliert mit der Tatsache, dass der Großteil der Ereignisse unbeobachtet erfolgt, in bis zu 90\% der Fälle primär ein nicht-defibrillierbarer Rhythmus vorliegt und vor allem sehr alte Patienten betrifft (Kitamura et al. 2014). Einzig in der erwähnten dänischen, allerdings retrospektiven Studie lag die Entlassrate mit ca. 9\% deutlich höher, wobei etwa die Hälfte der Patienten ein schlechtes neurologisches Ergebnis zeigte (Soholm et al. 2014).

Sehr hohes Alter ist bei einer Reanimation nicht der alleinige Prädiktor für ein negatives Outcome, aber ein wichtiger: Verglichen mit 65-79-Jährigen sinkt die Überlebenswahrscheinlichkeit nach den Ergebnissen einer prospektiven großen Beobachtungsstudie aus Japan bei 8o-89-Jährigen auf ein Drittel und bei über 9o-Jährigen auf ein Achtel. Der wichtigste Prädiktor für ein neurologisch intaktes Überleben war in jener Studie der initiale Herzrhythmus, wobei primäres Kammerflimmern die Überlebenswahrscheinlichkeit um den Faktor 16 steigerte. Weitere, demgegenüber jedoch deutlich weniger einflussreiche Faktoren waren die vor dem Ereignis verbliebenen Aktivitäten im täglichen Leben, das Ergreifen von Ersthelfermaßnahmen sowie die Eintreffzeit des Rettungsdienstes. Auch das Leben in einem Altenheim verschlechterte die Aussicht auf einen erfolgreichen Ausgang um den Faktor 2,5 (Kitamura et al. 2014). In der australischen Studie war die Prognose in Altenheimen bei nicht-defibrillierbaren Rhythmen etwa um den Faktor 4-8 schlechter als in allen anderen Lokalisationen des Herzstillstandes. Bei primärem Kammerflimmern war die Prognose im Vergleich zu häuslichen Herzstillständen allerdings nicht signifikant schlechter (Deasy et al. 2011). In Kopenhagen konnten die genannten Einflussfaktoren auf die Prognose ebenfalls nachgewiesen werden, mit insgesamt jedoch nicht derart ausgeprägten Einflüssen des Alters und des Herzrhythmus' (Soholm et al. 2014). Automatische externe Defibrillatoren könnten die Prognose von Patienten mit Kammerflimmern in Altenheimen weiter verbessern, sind jedoch hierzulande in diesen Einrichtungen die Ausnahme.

Bezüglich der Dauer der Reanimation empfiehlt der European Resuscitation Council (ERC), dass bei einer primären Asystolie die Reanimation nach 20 Minuten erfolgloser erweiterter Maßnahmen abgebrochen werden könne (Lippert et al. 2010), wobei allerdings jeder Fall individuell beurteilt werden müsse. Inwieweit eine solche Reanimation bei einem unbeobachteten asystolen Kreislaufstillstand eines schwer pflegebedürftigen Patienten medizinisch und ethisch Sinn macht, darf jedoch kritisch hinterfragt werden.

\section{Fallbeispiel:}

Der Notarzt wird zu einem 88-jährigen Patienten in ein Altenheim gerufen. Dieses wird nach ca. 13 Fahrminuten erreicht. Die RTW-Besatzung war ca. 4 Minuten zuvor eingetroffen und 
hatte den Mann leblos und asystol vorgefunden, worauf sie umgehend mit der kardiopulmonalen Reanimation begonnen hatte. Aktuelle Situation: Thoraxkompression und Beatmung über Larynxtubus erfolgen suffizient, es besteht weiterhin eine Asystolie. Der Notarzt lässt die Basisreanimation zunächst fortführen und erhebt parallel die Anamnese. Der Patient leidet seit vielen Jahren neben zahlreichen anderen Erkrankungen vor allem an einer Herzinsuffizienz im Stadium NYHA (New York Heart Association) IV mit zuletzt intermittierender $\mathrm{O}_{2}$-Abhängigkeit. Er hatte seit dem frühen Abend wieder vermehrt Luftnot empfunden und deswegen $\mathrm{O}_{2}$ erhalten. $\mathrm{Ca}$. 30 Minuten nach der letzten Visite sei er von der Pflegekraft leblos im Bett liegend vorgefunden worden. Reanimationsmaßnahmen waren bis zum Eintreffen des RTW nicht ergriffen worden. Eine Patientenverfügung liegt nicht vor. Angesichts des mutmaßlichen langen therapiefreien Intervalls und einer austherapierten Herzinsuffizienz bricht der Notarzt die Reanimation ab. Bei der Nachbesprechung äußert einer der beteiligten Rettungsassistenten sein völliges Unverständnis über die seiner Auffassung nach voreilige Beendigung der Reanimation. Eine weiterführende Erörterung über die Notwendigkeit, die Prognose der Grunderkrankung in Reanimationsentscheidungen einzubeziehen, lehnt er vehement und sichtbar frustriert ab. Der Notarzt nimmt die Situation zum Anlass, solche Szenarien in Zusammenarbeit mit dem Ärztlichen Leiter Rettungsdienst in einer aktuellen Fortbildungsveranstaltung zu thematisieren.

Fehlen gegenteilige Patientenverfügungen und liegt offensichtlich kein Terminalzustand vor, sollte insbesondere bei einem beobachteten Ereignis und/oder bei Kammerflimmern umgehend mit einer Reanimation begonnen werden. Im Fall, dass im Verlauf ein Terminalzustand oder der gegenteilige Patientenwille offensichtlich werden sollten, ist die Reanimation umgehend zu beenden. Prädiktoren für ein ungünstiges Reanimationsergebnis sind primärer Herzrhythmus, weit fortgeschrittene Grunderkrankung, massive Einschränkung in den Aktivitäten des täglichen Lebens, sehr hohes Alter, Unterlassen von Ersthelfermaßnahmen und ein Leben im Altenheim.

\subsection{Notfälle bei Pflegenden}

Wie einleitend erwähnt, werden über 1 Million Pflegebedürftige ausschließlich von ihren Angehörigen gepflegt - ganz überwiegend von der Ehefrau oder Tochter. Angesichts der enormen physischen und psychischen Belastung ist ein krankheitsbedingter Ausfall der Hauptpflegeperson ein Ereignis, mit dem jederzeit, besonders jedoch bei langjähriger Betreuung, gerechnet werden muss. In einem solchen Fall hat das Rettungsteam neben der medizinischen Versorgung des Patienten auch die Situation des Pflegebedürftigen zu bedenken und dessen weitere Versorgung zumindest anzustoßen. Findet sich nicht kurzfristig die Möglichkeit einer Überbrückung durch weitere Angehörige, Nachbarn oder einen ad hoc verfügbaren ambulanten Pflegedienst, bleibt u.U. keine andere Wahl, als den Patienten entweder akut in ein Heim (was häufig eine Herausforderung darstellt) oder in eine Klinik einzuweisen. Der Zusammenbruch des bisherigen Versorgungskonstrukts kann dabei für den Hilfsbedürftigen ein extrem traumatisierendes Erlebnis darstellen. 
6 Kritische Entscheidungssituationen und ethische Herausforderungen bei Notfällen in stationären und ambulanten Pflegeeinrichtungen

\section{Fallbeispiel:}

Mittwoch, 4:00 Uhr früh: Notarzteinsatz wegen „akutem Thoraxschmerz“. Eine 78-jährige Patientin berichtet, sie habe seit 3 Stunden Luftnot und einen wechselnden „Druck auf der Brust“. Im EKG zeigt sich ein akuter großer Vorderwandinfarkt. Klinisch besteht eine deutliche Lungenstauung. Nach Gabe von Sauerstoff, Morphin, Nitro, Furosemid, ASS und Heparin bessert sich der Zustand leicht. Als die Notärztin die Patientin telefonisch im Herzzentrum ankündigt, bittet diese, sie nicht sofort in die Klinik zu transportieren, da sie sich erst noch um die weitere Versorgung ihres pflegebedürftigen Ehemanns kümmern müsse. Dieser ist aufgrund einer langjährigen Parkinson-Erkrankung seit Jahren bettlägerig sowie harn- und stuhlinkontinent. Die Hauptpflegelast ruht auf der bislang körperlich gesunden Patientin. An Wochenenden wird sie im Wechsel von den weiter entfernt lebenden Kindern unterstützt. Die Notärztin erläutert der Frau die Notwendigkeit der sofortigen Katheterintervention. Jedoch gelingt es ihr erst, diese hiervon zu überzeugen, als sie die drohenden Folgen einer unterlassenen Behandlung für die weitere Versorgung des Ehemannes deutlich macht (Tod oder Invalidität, ohne die Möglichkeit, den Mann weiterhin versorgen zu können) und ihr verspricht, das Nötige zu dessen Versorgung zu veranlassen. Der telefonisch erreichte Sohn sichert das Kommen zu, wird jedoch erst in 3 Stunden eintreffen. Eine kurzzeitige Betreuung durch Nachbarn ist nicht möglich, ein ambulanter Pflegedienst kurzfristig nicht verfügbar. Aufgrund des Konflikts zwischen der dringlichen Transportnotwendigkeit und der fehlenden Betreuung des Ehemanns fordert die Notärztin einen weiteren RTW und den Ärztlichen Bereitschaftsdienst zur Klärung nach und tritt - bei inzwischen instabileren Kreislaufverhältnissen der Patientin - den Transport an. Nach Einsatzende erfährt sie, dass der Ehemann zwischenzeitlich psychisch völlig dekompensiert in eine nahe gelegene Neurologisch-Psychiatrische Fachklinik transportiert werden musste.

Ein Ausfall der häuslichen Hauptpflegeperson kann ein bislang reibungslos funktionierendes häusliches Pflegekonstrukt von einem Moment auf den anderen zerstören. Es ist daher keine unbillige Forderung, für unsere alternde Gesellschaft neben dem Rettungsdienst auch einen sog. „Pflegenotdienst", zumindest jedoch ausreichend Akutpflegeplätze in Heimen zu fordern.

\subsection{Fazit und Ausblick}

Einsätze bei pflegebedürftigen Patienten stellen ein zunehmendes und in mehrfacher Hinsicht sehr anspruchsvolles Feld der Notfallmedizin dar. Probleme der Entscheidungsfindung bei Notfällen mit pflegebedürftigen Patienten sind insbesondere:

- Unkenntnis der Biographie

- Unsicherheit hinsichtlich der Prognose der aktuellen Erkrankung und von Vorerkrankungen

- Unkenntnis bzw. schwierige Interpretation des Patientenwillens

- hohen Zeitdruck

v u.U. zum Patientenwillen widersprüchliche Erwartungshaltung von Angehörigen und/oder Dritten

- beträchtliche Gefahr von Fehlentscheidungen

- Grenzen von Algorithmen für die individuelle Entscheidungsfindung 
Die rückläufige Präsenz von Hausärzten und Notärzten in der Fläche stellt vor diesem Hintergrund eine Entwicklung dar, deren Folgen auf die Versorgung dieser Patienten noch gar nicht richtig abschätzbar sind. Die zukünftig bessere Ausbildung des Rettungsfachpersonals - auch in Bezug auf geriatrische Patienten - im Rahmen des Notfallsanitätergesetzes ist fachlich uneingeschränkt zu begrüßen. Gleichzeitig muss betont werden, dass die Entscheidungsfindung in medizinischen, ethischen und rechtlichen Grenzsituationen eine extreme Herausforderung darstellen kann, die nicht in einfachen Algorithmen abbildbar ist, ein beträchtliches Risiko von Fehlentscheidungen beinhaltet und daher aus gutem Grund ärztliche Kernaufgabe bleiben muss.

Nach Redaktionsschluss sind die neuen Reanimationsleitlinien des European Resuscitation Council (ERC) erschienen, inkl. eines neuen Kapitels zur Ethik der Reanimation und Entscheidungen am Lebensende. Die deutsche Übersetzung ist über die Homepage des German Resuscitation Council (GRC) mit dem folgenden Link kostenfrei zugänglich: http://www. grc-org.de/leitlinien2015. Gegenüber der Fassung von 2010 sind insbesondere die Aspekte Patientenautonomie und -rechte, Fürsorge, Schadensvermeidung, Gerechtigkeit, Aussichtslosigkeit, Vorausverfügungen sowie Unterschiede in der ethischen Praxis in Europa deutlich ausführlicher bzw. z.T. erstmalig dargestellt. Die ergänzende Lektüre wird empfohlen.

\section{Literatur}

Beauchamp TL, Childress JF (2012) Principles of biomedical ethics. 7. Auflage. Oxford University Press New York

Benetos A, Labat C, Rossignol P, Fay R, Rolland Y, Valbusa F, Salvi P, Zamboni M, Manckoundia P, Hanon 0, Gautier S (2015) Treatment with multiple blood pressure medications, achieved blood pressure, and mortality in older nursing home residents: The PARTAGE Study. JAMA Intern Med 16 [Epub ahead of print]

Birnbacher D, Klitsch W, Langenberg U, Barnikol UB (2015) Umgang mit Demenzpatienten: Gemeinsam verantwortete Entscheidungen. Dtsch Arztebl 112: 514-515

Brandt HE, Delines L, Ooms ME, can der Steen JT, can der Wal G, Ribbe MW (2005) Symptoms, signs, problems and diseases of terminally ill nursing home patients. Arch Intern Med 165: 314-320

Bundesministerium für Gesundheit (2011) Fünfter Bericht der Bundesregierung über die Entwicklung der Pflegeversicherung und den Stand der pflegerischen Versorgung in der Bundesrepublik Deutschland. http://www. bmg.bund.de/fileadmin/dateien/Publikationen/Pflege/Berichte/Bericht_der_Bundesregierung_ueber_ die_Entwicklung_der_Pflegeversicherung_und_den_Stand_der_pflegerischen_Versorgung_in_der_Bundesrepublik_Deutschland.pdf (Zugriff am 24.03.2015)

Deasy C, Bray JE, Smith K, Harriss LR, Bernard SA, Cameron P, VACAR Steering Committee (2011) Out-of-hospital cardiac arrests in the older age groups in Melbourne, Australia. Resuscitation 82: 398-403

Hoffmann F, Kaduszkiewicz H, Glaeske G, van den Bussche H, Koller D (2014) Prevalence of dementia in nursing home and community-dwelling older adults in Germany. Aging Clin Exp Res 26: 555-559

Kitamura T, Morita S, Kiyohara K, Nishiyamad C, Kajinoe K, Sakaie T, Nishiuchif T, Hayashib Y, Shimazue T, Iwamig T (2014) Trends in surviving among elderly patients with out-of-hospital cardiac arrest: a prospective, population-based observation from 1999 to 2011 in Osaka. Resuscitation 85: 1432-1438

Lippert FK, Raffay V, Georgiou M, Steen PA, Bossaert L (2010) Ethik der Reanimation und Entscheidungen am Lebensende. Sektion 10 der Leitlinien zur Reanimation 2010 des European Resuscitation Council. Notfall Rettungsmed 13: 737-744

Luiz T, Madler C (2009) Einsätze in Altenheimen. Notfallmedizin up2date 4: 313-328

McParland E, Likourezos A, Chichin E, Castor T, Paris BE (2003) Stability of preferences regarding life-sustaining treatment: a two-year prospective study of nursing home residents. Mt Sinai I Med 70: 85-92

Parry R, Seymour I, Whittaker B, Bird L, Cox K (2013) Rapid evidence review: pathways focused on the dying phase in end of life care and their key components. http://socialwelfare.bl.uk/subject-areas/services-activity/healthservices/departmentofhealth/150893review_academic_literature_on_end_of_life.pdf (Zugriff am 15.04.2015) 
6 Kritische Entscheidungssituationen und ethische Herausforderungen bei Notfällen in stationären und ambulanten Pflegeeinrichtungen

Prückner S, Madler C (2009) Der demographische Wandel. Notfallmedizin für eine alternde Gesellschaft. Notfall Rettungsmed 12: 13-18

Soholm H, Bro-Jeppesen I, Lippert FK, Køber L, Wanscher M, Kjaergaard I, Hassager C (2014) Resuscitation of patients suffering from sudden cardiac arrests in nursing homes is not futile. Resuscitation 85: 369-375

Statistisches Bundesamt (2013) Pflegestatistik. Pflege im Rahmen der Pflegeversicherung. Deutschlandergebnisse. https://www.destatis.de/DE/Publikationen/Thematisch/Gesundheit/Pflege/PflegeDeutschlandergebnisse. html;jsessionid=D8DC4EFEFBFDD73A4B999690312AEgB9.cae2 (Zugriff am 24.03.2015)

Wiese CHR, Duttge G, Weber AK, Zausig YA, Ruppert D, Hanekop GG, Graf BM (2009) Notfallmedizinische Betreuung von Palliativpatienten am Lebensende. Anaesthesist 58: 1097-1106

Wiese CHR, Zausig YA, Vormelker I, Orso S, Graf BM, Hanekop GG (2010) Ambulante und stationäre palliativmedizinische Patientenversorgung in Deutschland. Anaesthesist 59: 162-170

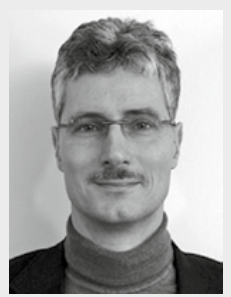

\section{Dr. med. Thomas Luiz}

1982-1988 Studium der Medizin an der Universität Heidelberg. 1998-1995 Weiterbildung zum Arzt für Anästhesie am Theresien-Krankenhaus Mannheim sowie am Universitätsklinikum Mannheim. 1999 Wechsel an die Klinik für Anästhesie und Notfallmedizin am Westpfalz-Klinikum Kaiserslautern. Seit 2000 Oberarzt. 2005-2008 Ärztlicher Leiter Rettungsdienst. Seit 2009 Projektleiter Medizin am Deutschen Zentrum für Notfallmedizin \& IT am Fraunhofer IESE. Forschungsschwerpunkte: Epidemiologie psychosozialer und geriatrischer Notfälle, Ambiente Notfallerkennung, logistische Aspekte der Notfallmedizin, Epidemiologie und Therapie des Herzkreislaufstillstandes. 